\title{
GROUP ACTIONS AND DEFORMATIONS FOR HARMONIC MAPS INTO SYMMETRIC SPACES
}

\author{
BY Yoshihiro OHNITA
}

Let $M$ and $N$ be Riemannian manifolds. The energy of a smooth map $\varphi: M \longrightarrow N$ is defined as

$$
E(\varphi)=\frac{1}{2} \int_{M}\|d \varphi\|^{2} d v
$$

A smooth map $\varphi$ is called harmonıc if the first variation of the energy vanishes for every smooth variation of $\varphi$ with compact support. In the case $\operatorname{dim} M=2$, since the energy is invariant under conformal deformations of the Riemannian metric on $M$, it is natural to consider a Riemann surface $\Sigma$ rather than a Riemannian manifold $M$ as the domain manifold.

This article is concerned with two related areas of harmonic map theory ; group actions and deformations for harmonic maps of Riemann surfaces $\Sigma$ into symmetric spaces $N$.

The action of a certain infinite dimensional Lie group and algebra on harmonic maps from a simply connected Riemann surface, especially a Riemann sphere, into a compact Lie group or a symmetric space have been investigated by Uhlenbeck [Uh], Zakharov-Shabat-Mikhailov [ZM,ZS] others. In a joint paper with M.A. Guest ([GO1]), we have shown how the action of a infinite dimensional Lie group can be interpreted in terms of the Grassmannian model in Loop Group Theory (cf. [PS],[Se]) and investigated its geometric nature, and as an important application we discussed deformations of harmonic maps from the viewpoint of Morse-Bott theory over twistor spaces. Using this idea, we have given results on the connectedness of spaces of harmonic 2-spheres in the standard sphere $S^{n}$, the real projective space $\mathbf{R} P^{n}$ (see also [Ko]) and the complex projective space $\cdot \mathbf{C} P^{n}$, the unitary group $U(n)$, and in [FGKO] we have determined the fundamental group of the space of harmonic 2-spheres in $S^{n}$.

In Section 1 and 2 we shall review the construction of extended solutions of harmonic maps into Lie groups and the natural action of the complex loop group on harmonic maps into Lie groups. In Section 3 we shall discuss group actions on harmonic maps into symmetric spaces of inner type and in Section 4 we shall mention further results on the connectedness of certain spaces of harmonic 2-spheres in symmetric spaces. These are joint works with M.A. Guest and M.Mukai in progress.

\section{Extended solutions for harmonic maps}

Let us begin with the definition of the notion of extended solutions of harmonic maps into Lie groups. Let $G$ be a compact connected Lie group and $\mathfrak{g}$ be its Lie algebra. We

Received October 15, 1993. 
equip $G$ with a biinvariant Riemannian metric. Then $G$ becomes a compact Riemannian symmetric space. Denote by $\mu=\mu_{G}$ the (left invariant) Maurer-Cartan form of $G$, which satisfies the Maurer-Cartan equation

$$
d \mu+\frac{1}{2}[\mu \wedge \mu]=0 .
$$

Now let $\varphi: \Sigma \longrightarrow G$ be a smooth map of a Riemann surface $\Sigma$ into $G$. Set $\alpha=\varphi^{*} \mu=$ $\varphi^{-1} d \varphi$, which is a 1 -form with values in $g$. Then it satisfies

$$
d \alpha+\frac{1}{2}[\alpha \wedge \alpha]=0 \text {. }
$$

We shall decompose the 1 -form $\alpha$ into $\alpha=\alpha^{\prime}+\alpha^{\prime \prime}$, where $\alpha^{\prime}$ and $\alpha^{\prime \prime}$ denote the $(1,0)$ part and $(0,1)$-part of $\alpha$ with respect to the complex structure of $\Sigma$. It is known (cf. [Uh]) that the map $\varphi$ is harmonic if and only if

$$
\bar{\partial} \alpha^{\prime}-\partial \alpha^{\prime \prime}=0 \text {. }
$$

Let $\Omega G=\left\{\gamma: S^{1} \longrightarrow G\right.$ smooth,$\left.\gamma(1)=e\right\}$ be the group of based smooth loops in $G$. (The completion of) $\Omega G$ has the standard infinite dimensional complex manifold structure and Kähler structure (cf. [PS]). We identify the complexified tangent space $T_{e} \Omega G^{\mathbf{C}}$ with the based loop algebra $\Omega \mathfrak{g}^{\mathbf{C}}=\bigoplus_{\alpha \neq 0}\left(\lambda^{\alpha}-1\right) \mathfrak{g}^{\mathbf{C}}$. The (left-invariant) complex structure is defined as

$$
T_{e} \Omega G^{1,0}=\bigoplus_{\alpha>0}\left(\lambda^{-\alpha}-1\right) \mathfrak{g}^{\mathbf{C}} .
$$

Define a surjective map $\pi: \Omega G \longrightarrow G$ by $\pi(\gamma)=\gamma(-1)$

We call an extended solution (of a harmonic map) a smooth map $\Phi: \Sigma \longrightarrow \Omega G$ satisfying the condition

$$
\Phi^{*} \mu_{\Omega G}\left(T \Sigma^{1,0}\right)=\Phi^{-1} d \Phi\left(T \Sigma^{1,0}\right) \subset\left(\lambda^{-1}-1\right) g^{\mathbf{C}}
$$

We should remark that an extended solution $\Phi: \Sigma \longrightarrow \Omega G$ is a holomorphic map relative to the above standard complex structure of $\Omega G$.

THEOREM $1.1[\mathrm{Po}, \mathrm{Uh}, \mathrm{ZS}, \mathrm{ZM}]$.

(1) If $\Phi: \Sigma \longrightarrow \Omega G$ is an extended solution, then $\varphi=\pi \circ \Phi: \Sigma \longrightarrow G$ us harmonıc.

(2) Assume that $\Sigma$ is simply connected. Let $\varphi: \Sigma \longrightarrow G$ be a harmonic map. For $z_{0} \in \Sigma$ and $\delta \in \Omega G$, there is a unıque extended solution $\Phi: \Sigma \longrightarrow \Omega G$ such that $\Phi\left(z_{0}\right)=\delta$ and $\pi \circ \Phi=c \varphi$ for $c \stackrel{=}{=}(-1) \varphi\left(z_{0}\right)^{-1} \in G$.

This fact is obtained as follows : assume that $\alpha=\alpha^{\prime}+\alpha^{\prime \prime}$ is a $\mathrm{g}$-valued 1-form on $\Sigma$, where $\alpha^{\prime}$ and $\alpha^{\prime \prime}$ are the $(1,0)$-part and $(0,1)$-part of $\alpha$, respectively. For each $\lambda \in S^{1}=\{\lambda \in \mathbf{C}|| \lambda \mid=1\}$, define

$$
\alpha_{\lambda}=\frac{1}{2}\left(1-\lambda^{-1}\right) \alpha^{\prime}+\frac{1}{2}(1-\lambda) \alpha^{\prime \prime}
$$

Consider the linear partial differential equation of the first order : for each $\lambda \in S^{1}$,

$$
\Phi_{\lambda}^{*} \mu_{G}=\alpha_{\lambda},
$$

or equivalently

$$
\left\{\begin{array}{l}
\partial \Phi_{\lambda}=\frac{1}{2}\left(1-\lambda^{-1}\right) \Phi_{\lambda} \alpha^{\prime} \\
\bar{\partial} \Phi_{\lambda}=\frac{1}{2}(1-\lambda) \Phi_{\lambda} \alpha^{\prime \prime}
\end{array}\right.
$$


Then the important observation is that the complete integrability condition of $(*)$ or $(* *)$

$$
d \alpha_{\lambda}+\frac{1}{2}\left[\alpha_{\lambda} \wedge \alpha_{\lambda}\right]=0 \text { for all } \lambda \in S^{1}
$$

is equivalent to (1) and (2). Therefore we obtain the above theorem.

Assume that $G=U(n)$. If a harmonic map $\varphi: \Sigma \longrightarrow U(n)$ admits an extended solution $\Phi: \Sigma \longrightarrow \Omega U(n)$ with $\pi \circ \Phi=a \varphi$ for some $a \in U(n)$ which has finite Fourier expansion

$$
\Phi=\sum_{\alpha=0}^{m} \lambda^{\alpha} T_{\alpha}
$$

then we say that $\varphi$ has finte uniton number. Such a minimal number $m$ is called the minimal uniton number of $\varphi$. This concept was introduced in [Uh] and she proved that harmonic maps of finite uniton number can be factorized into so called unitons. This shows how such harmonic maps may be constructed from holomorphic maps into complex Grassmann manifolds. It is known (see [OV]) that a harmonic map of finite uniton number is weakly conformal and thus a branched minimal immersion. Uhlenbeck [Uh] showed that any harmonic map $\varphi: S^{2} \longrightarrow U(n)$ always has finite uniton number.

\section{Loop group actions on harmonic maps}

First we recall the notion of the Grassmannian model of $\Omega G$ (Quillen, [PS]).

Let $G$ be a compact connected Lie group with trivial center with Lie algebra $\mathfrak{g}$. The Grassmannian model of $\Omega G$ is defined as follows ([PS]) : we consider $H=H^{\mathfrak{g}}=$ $L^{2}\left(S^{1}, \mathfrak{g}^{\mathbf{C}}\right)$, which has a complex Lie algebra structure of infinite dimension. The loop groups $L G^{\mathbf{C}}$ and $L G$ act on $H^{\mathfrak{g}}$ via the adjoint representation. We shall decompose $H=H_{+} \oplus H_{-}$, where $H_{+}$is the closed subspace of all functions with Fourier expansion $f=\sum_{k \geq 0} \lambda^{k} a_{k}$ and $H_{-}=\left(H_{+}\right)^{\perp}$. Define

$$
G r_{\infty}^{\mathfrak{g}}=\left\{W \in G r_{\infty} \mid \bar{W}^{\perp}=\lambda W, W^{s m} \text { is a Lie algebra }\right\}
$$

Here $W^{s m}$ denotes the subspace of smooth functions in $W$. Then the loop groups $L G^{\mathbf{C}}$ and $L G$ act transitively on $G r_{\infty}^{\mathfrak{g}}$ and their isotropy groups at $H_{+} \in G r_{\infty}^{\mathfrak{g}}$ are $L^{+} G^{\mathbf{C}}$ and $G$, respectively. 'Thus we get the identifications

$$
G r_{\infty}^{\mathfrak{g}} \cong L G^{\mathbf{C}} / L^{+} G^{\mathbf{C}} \cong L G / G \cong \Omega G \text {. }
$$

Then the diffeomorphism $\Omega G \longrightarrow G r_{\infty}^{\mathfrak{g}}$ is given by $\gamma \longmapsto \gamma H_{+}$. Hence we have the following fact which is referred to the Iwasawa decomposition for loops ([PS]) : each $\gamma \in L G^{\mathrm{C}}$ can be uniquely decomposed as

where $\gamma_{u} \in \Omega G$ and $\gamma_{+} \in L^{+} G^{\mathbf{C}}$.

$$
\gamma=\gamma_{u} \gamma_{+}
$$

Let $W: \Sigma \longrightarrow G r_{\infty}$ be the map corresponding to a smooth map $\Phi: \Sigma \longrightarrow \Omega G$ by $W(z)=\Phi(z) H_{+}$for all $z \in \Sigma$. Then $\Phi$ is a holomorphic map if and only if $W$ satisfies

$$
\frac{\partial}{\partial \bar{z}} C^{\infty}(W) \subset C^{\infty}(W)
$$

Moreover, a holomorphic map $\Phi$ is an extended solution if and only if $W$ satisfies

$$
\frac{\partial}{\partial z} C^{\infty}(W) \subset \lambda^{-1} C^{\infty}(W)
$$


Definition. The natural action of $L G^{\mathbf{C}}$ on $\Omega G$ is defined as follows : for each $\gamma \in L G^{C}$ and each $\delta \in \Omega G$, then

$$
\gamma^{\natural} \delta=\gamma \delta(\gamma \delta)_{+}^{-1}=(\gamma \delta)_{u}
$$

or equivalently

$$
\gamma^{\natural} \delta H_{+}=\gamma \delta H_{+}
$$

on the Grassmannian model.

For any $\gamma \in L G^{\mathbf{C}}$ and any extended solution $\Phi: \Sigma \longrightarrow \Omega G$, we define a map $\gamma^{\natural} \Phi: \Sigma \longrightarrow \Omega G$ by $\left(\gamma^{\natural} \Phi\right)(z)=(\gamma \Phi(z))_{u}$, or equivalently in terms of the Grassmannian model $\left(\gamma^{\natural} \Phi\right)(z) H_{+}=\gamma \Phi(z) H_{+}$, for each $z \in \Sigma$. Then it was shown in [GO1] that the $\operatorname{map} \gamma^{\natural} \Phi: \Sigma \longrightarrow \Omega G$ is an extended solution. Hence we have

THEOREM 2.1 [GO1]. Assume that $\Sigma$ is a simply connected Riemann surface. Then the complex loop group $L G^{\mathbf{C}}$ acts on the space of harmonıc maps $\varphi: \Sigma \longrightarrow G$.

The $S^{1}$-action $\sharp$ on $\Omega G$ (cf. [Uh]) is defined as

$$
\left(v^{\sharp} \gamma\right)(\lambda)=\gamma\left(v^{-1} \lambda\right) \gamma\left(v^{-1}\right)^{-1}
$$

for each $v \in S^{1}$ and each $\gamma \in \Omega G$. This $S^{1}$-action $\sharp$ preserves the standard complex Kähler manifold structure of $\Omega G$.

\section{Group actions on harmonic maps into symmetric spaces}

We shall discuss actions of the complex loop group on harmonic maps into compact symmetric spaces of inner type.

Let $G$ be a compact connected Lie group with trivial center with Lie algebra $\mathfrak{g}$. Assume that $M=G / K$ is a compact symmetric space with symmetric pair $(G, K, \sigma)$, where $\sigma$ is an involutive automorphism of $G$ and $\left(G_{\sigma}\right)^{0} \subset K \subset G_{\sigma}$, where $G_{\sigma}$ and $\left(G_{\sigma}\right)^{0}$ are the subgroup of all fixed elements by $\sigma$ and its identity component, respectively. Define the subgroups of $L G$ and $L G$ by

$$
L\left(G^{\mathbf{C}}, \sigma\right)=\left\{\gamma \in L G^{\mathbf{C}} \mid \sigma(\gamma(\lambda))=\gamma(-\lambda) \text { for all } \lambda \in S^{1}\right\}
$$

and $L(G, \sigma)=L G \cap L\left(G^{\mathbf{C}}, \sigma\right)$. Then we have

Theorem 3.1 [GO2]. Assume that $\Sigma$ is a simply connected Rremann surface. Then the complex loop group $L\left(G^{\mathbf{C}}, \sigma\right)$ acts on the space of harmonic maps $\varphi: \Sigma \longrightarrow G / K$.

Here, in the case where $M=G / K$ is a symmetric space of inner type, we introduce a loop group action different from above.

Set $N=\left\{a \in G \mid a^{2}=e\right\}$. Each connected component of $N$ is a totally geodesic submanifold of even dimension, which is called a polar of $G$ and was investigated by $\mathrm{T}$. Nagano. They are compact symmetric spaces of inner type and all compact symmetric spaces of inner type can be obtained in this way. Now we define the subgroups of $L G^{\mathbf{C}}$ and $L G$ as

$$
L^{\prime} G^{\mathbf{C}}=\left\{\gamma \in L G^{\mathbf{C}} \mid \gamma(\lambda)=\gamma(-\lambda) \text { for all } \lambda \in S^{1}\right\}
$$


and

$$
\begin{aligned}
L^{\prime} G & =L G \cap L^{\prime} G^{\mathbf{C}}, \\
L^{+,} G & =L^{+} G \cap L^{\prime} G^{\mathbf{C}} .
\end{aligned}
$$

We define a loop subspace of $\Omega G$ by

$$
\Omega^{\prime} G=\left\{\gamma \in \Omega G \mid \gamma(\lambda)=\gamma(-\lambda) \gamma(-1)^{-1} \text { for all } \lambda \in S^{1}\right\} .
$$

The surjective map $\pi: \Omega^{\prime} G \longrightarrow N$ is defined as the restriction of $\pi: \Omega G \longrightarrow G$. Set

$$
G r_{\infty}^{\mathfrak{g}, \boldsymbol{\prime}}=\left\{W \in G r_{\infty}^{\mathfrak{g}} \mid \tau(W)=W\right\}
$$

where $\tau(f)(\lambda)=f(-\lambda)$. Then we have

$$
G r_{\infty}^{\mathfrak{g}, \prime} \cong L^{\prime} G^{\mathbf{C}} / L^{+, \prime} G^{\mathbf{C}} \cong L^{\prime} G / G \cong \Omega^{\prime} G
$$

Assume that $\varphi: \Sigma \longrightarrow N$ is a harmonic map of a simply connected Riemann surface $\Sigma$ into the symmetric space $N$. For each $\delta \in \Omega^{\prime} G$ with $\pi(\delta)=\delta_{-1}=\varphi\left(z_{0}\right)$, by Theorem 1.1 there is an extended solution $\Phi: \Sigma \longrightarrow \Omega G$ such that $\Phi\left(z_{0}\right)=\delta$. Then we can show that $\varphi=\pi \circ \Phi$ and the image of $\Phi$ is contained in $\Omega^{\prime} G$. This was proved by [Uh] in the case of $G=U(n)$. Hence we have

THEOREM 3.2. Assume that $\Sigma$ is a simply connected Riemann surface. Then the complex loop group $L^{\prime} G^{\mathbf{C}}$ acts on the space of harmonic maps $\varphi: \Sigma \longrightarrow N$.

In this case it is possible to construct finite dimensional twistor subspaces of $\Omega^{\prime} G$. Consider

$$
\mathcal{F}=\left\{\omega \in \Omega^{\prime} G \mid \omega(\lambda \mu)=\omega(\lambda) \omega(\mu) \text { for all } \lambda, \mu \in S^{1}\right\},
$$

which is the set of all fixed points under the $S^{1}$-action $\sharp$. Thus each connected component of $\mathcal{F}$ is a totally geodesic, complex submanifold of $\Omega G$ (see [EL]). Let $\omega \in \mathcal{F}$. There is an element $P$ in $\mathfrak{g}$ with $\exp (t P)=\omega(\lambda)$, where $\lambda=e^{2 \pi \sqrt{-1} t}$. Then we have a generalized flag manifold $G / C_{P}$, where $C_{P}$ is a centralizer in $G$ of $P$. We have the identification

$$
C_{P}=\left\{a \in G \mid A d(a) \omega(\lambda)=\omega(\lambda) \text { for each } \lambda \in S^{1}\right\}
$$

and

$$
G / C_{P}=\left\{\operatorname{Ad}(a) \omega \in \Omega^{\prime} G \mid a \in G\right\} .
$$

Let $K=\{a \in \dot{G} \mid A d(a) \omega(-1)=\omega(-1)\}$. Then we have a compact symmetric space $G / K$ totally geodesically embedded in $G$, which is a connected component of $N$. The linear endomorphism $a d(P)$ on $\mathfrak{g}^{\mathbf{C}}$ has eigenvalues in $2 \pi \sqrt{-1} \mathbf{Z}$ and we denote by $\mathfrak{g}_{\ell}$ the $2 \pi \sqrt{-1} \ell$-eigenspace of $a d(P)$. We have the eigenspace decomposition of $g^{\mathbf{C}}$ with respect to $\operatorname{ad}(P)$ :

$$
\mathfrak{g}^{\mathbf{C}}=\bigoplus_{\ell} \mathfrak{g}_{\ell}
$$

Then we have

$$
\mathfrak{c}_{P}^{\mathbf{C}}=\mathfrak{g}_{0} \quad \text { and } \quad \mathfrak{k}^{\mathbf{C}}=\bigoplus_{\ell \text { even }} \mathfrak{g}_{\ell},
$$

where $c_{P}$ and $\mathfrak{k}$ denote the Lie algebras of $C_{P}$ and $K$, respectively. We have the natural projection $\pi: G / C_{P} \longrightarrow G / K$ as a restriction of $\pi: \Omega G \longrightarrow G$. This fibration was investigated by $[\mathrm{BR}]$ from the viewpoint of twistor geometry and applications to harmonic 
maps. Relative to the fibration $\pi: G / C_{P} \longrightarrow G / K$, the complexified vertical subspace corresponds to $\bigoplus_{\ell} \neq 0$ even $g_{\ell}$ and the complexified horizontal subspace corresponds to $\bigoplus_{\ell}$ odd $\mathfrak{g}_{\ell}$. The super-horizontal subspace ([BR]) corresponds to $\mathfrak{g}_{-1} \oplus \mathfrak{g}_{1}$. Let $K^{\mathbf{C}}$ be a complex Lie subgroup of $G^{\mathbf{C}}$ generated by $\mathfrak{k}^{\mathbf{C}}$. It is known that if $f: \Sigma \longrightarrow G / C_{P}$ is horizontal and holomorphic, then $\pi \circ f: \Sigma \longrightarrow G / K$ is harmonic (cf. [Ra,Sa]).

Under the identifications $T_{e C_{P}} G / C_{P}^{\mathbf{C}} \cong \bigoplus_{\ell \neq 0} \mathfrak{g}_{\ell}$ and $T_{\omega} \Omega G^{\mathbf{C}} \cong T_{e} \Omega G^{\mathbf{C}} \cong \bigoplus_{\ell \neq 0} \mathbf{g}_{\ell}$, the derivative at the origin $e C_{P}$ of the embedding $\iota: G / C_{P} \longrightarrow \Omega^{\prime} G \subset \Omega G$ identifies $\mathfrak{g}_{\ell}$ with $\left(\lambda^{-\ell}-1\right) \mathfrak{g}_{\ell}$ (e.g. see [GO1]). Hence we see that if $f: \Sigma \longrightarrow G / C_{P}$ is superhorizontal and holomorphic, then $\iota \circ f$ is an extended solution as a smooth map into $\Omega G$.

Let $G_{P}$ be the parabolic subgroup of $G^{\mathbf{C}}$ generated by a parabolic subalgebra $\mathbf{g}_{P}=\bigoplus_{\ell \leqq 0} \mathfrak{g}_{\ell}$. Then it is well-known that $G / C_{P}=G^{\mathbf{C}} / G_{P}$ and thus it becomes a complex manifold. The embedding $\iota: G / C_{P}=G^{\mathbf{C}} / G_{P} \longrightarrow \Omega^{\prime} G=L^{\prime} G^{\mathbf{C}} / L^{+,{ }^{\prime}} G^{\mathbf{C}}$ is holomorphic and $G^{\mathbf{C}}$-equivariant with respect to the injective homomorphism $G^{\mathbf{C}} \longrightarrow L^{\prime} G^{\mathbf{C}}$ of constant loops. Then the action of $G^{\mathrm{C}}$ on $G / C_{P}$ preserves the holomorphicity condition and the super-horizontality condition, and the action of $K^{\mathbf{C}}$ on $G / C_{P}$ preserves the holomorphicity condition and the horizontality condition (see [GO2]). But, in general, the action of $G^{\mathbf{C}}$ on $G / C_{P}$ does not preserve the horizontality condition. We can characterize the twistor fibrations such that the action of $G^{\mathrm{C}}$ on $G / C_{P}$ preserves the horizontality condition.

Obviously, if $\mathfrak{g}_{\ell}=0$ for $|\ell|>2$, the concepts of horizontality and super-horizontality coincide and hence the action of $G^{\mathbf{C}}$ on $G / C_{P}$ preserves the horizontality condition. In this case, $a d P$ gives a gradation of this type

$$
\mathfrak{g}^{\mathbf{C}}=\mathfrak{g}_{-2}+\mathfrak{g}_{-1}+\mathfrak{g}_{0}+\mathfrak{g}_{1}+\mathfrak{g}_{2} .
$$

Such twistor fibrations over symmetric spaces were investigated and classified by [Br] and [Sa]. These twistor fibrations form a distinguished class of twistor fibrations over symmetric spaces. We call such a twistor fibration $\pi: Z \longrightarrow M$ over $M$ standard and the twistor space $Z$ over a symmetric space $M$ the standard twrstor space of $M$.

Proposition 3.3 (see [GO2]). The action of $G^{\mathrm{C}}$ on $G / C_{P}$ preserves the horizontal subspaces if and only if the twistor fibration $G / C_{P} \longrightarrow G / K$ is isomorphic to the standard twistor fibration over $M=G / K$.

See $[\mathrm{Br}],[\mathrm{Sa}]$ for the complete list of the standard twistor spaces. The following is a concrete description for all standard twistor spaces $Z$ over each classical symmetric space $M$ of inner type. In this list we use the following notation. Let $\langle$,$\rangle be the standard$ Hermitian inner product on $\mathbf{C}^{N}$ defined by $\langle u, v\rangle=\sum_{i=1}^{N} u_{i} \bar{v}_{i}$. We define the complex symmetric bilinear form ( , ) on $\mathbf{C}^{N}$ by $(u, v)=\langle u, \bar{v}\rangle$. Let $J: \mathbf{C}^{2 N} \longrightarrow \mathbf{C}^{2 N}$ denote the conjugate linear map corresponding to the left multiplication of $j$ under the identification $\mathbf{H}^{N} \cong \mathbf{C}^{2 N}$ by $\left(z_{1}+z_{N+1} j, \ldots, z_{N}+z_{2 N} j\right) \longmapsto\left(z_{1}, \ldots, z_{N}, z_{N+1}, \ldots, z_{2 N}\right)$. We define the complex symmetric bilinear form $(,)^{a}$ on $\mathbf{H}^{N} \cong \mathbf{C}^{2 N}$ by $(u, v)^{a}=\langle u, J(v)\rangle$.

(AIII) In the case $M=G r_{k}\left(\mathbf{C}^{N}\right)$,

(1) $Z=M=G r_{k}\left(\mathbf{C}^{N}\right)$ and $\pi=i d$, 
(2) for $1 \leqq p<N-k$,

$$
\begin{aligned}
Z & =\mathcal{F}_{p, p+k}\left(\mathbf{C}^{N}\right) \\
& =\left\{\left(W_{p}, W_{p+k}\right) \in G r_{p}\left(\mathbf{C}^{N}\right) \times G r_{p+k}\left(\mathbf{C}^{N}\right) \mid W_{p} \subset W_{p+k}\right\}
\end{aligned}
$$

and $\pi\left(W_{p}, W_{p+k}\right)=W_{p}^{\perp} \cap W_{p+k}$

(3) for $1 \leqq p<k$ with $k=p+q$,

$$
\begin{aligned}
& Z=\mathcal{F}_{p, q}^{\perp}\left(\mathbf{C}^{N}\right) \\
& =\left\{\left(W_{p}, W_{N-q}\right) \in G r_{p}\left(\mathbf{C}^{N}\right) \times G r_{N-q}\left(\mathbf{C}^{N}\right) \mid W_{p} \subset W_{N-q}\right\} \\
& \text { and } \pi\left(W_{p}, W_{N-q}\right)=W_{p} \oplus W_{N-q}^{\perp} \text {. }
\end{aligned}
$$

(BDI) In the case $M=\widetilde{G r}_{k}\left(\mathbf{R}^{N}\right)$,

(1) for $k=2 m$ and $\ell$ odd with $N=k+\ell$,

$$
Z=\mathcal{Z}_{m}\left(\mathbf{C}^{N}\right)=\left\{W \in G r_{m}\left(\mathbf{C}^{N}\right) \mid(W, W)=0\right\}
$$

and $\pi(W)=W \oplus \bar{W}$

(2) for $k=2 m$ and $\ell=2 n$ with $N=k+\ell$,

$$
Z=\mathcal{Z}_{m}\left(\mathbf{C}^{N}\right)=\left\{W \in G r_{m}\left(\mathbf{C}^{N}\right) \mid(W, W)=0\right\}
$$

and $\pi(W)=W \oplus \bar{W}$

(3) for $k=2 m$ and $\ell=2 n$ with $N=k+\ell$,

$$
Z=\mathcal{Z}_{n}\left(\mathbf{C}^{N}\right)=\left\{W \in G r_{n}\left(\mathbf{C}^{N}\right) \mid(W, W)=0\right\}
$$

and $\pi(W)=(W \oplus \bar{W})^{\perp}$.

(CI) In the case $M=S p(n) / U(n)=\left\{V \in G r_{n}\left(\mathbf{C}^{2 n}\right) \mid(V, V)^{a}=0\right\}$,

$$
Z=M=S p(n) / U(n) \text { and } \pi=i d \text {. }
$$

(CII) In the case $M=G r_{k}\left(\mathbf{H}^{N}\right)=\left\{V \in G r_{2 k}\left(\mathbf{C}^{2 N}\right) \mid J(V)=V\right\}$,

(1) for $k+\ell=N, \quad Z=\mathcal{T}_{\ell}\left(\mathbf{C}^{2 N}\right)$ and $\pi(W)=(W \oplus J W)^{\perp}$

$$
=\left\{W \in G r_{\ell}\left(\mathbf{C}^{2 N}\right) \mid(W, W)^{a}=0\right\}
$$

(2) for $1 \leqq k<N, \quad Z=\mathcal{T}_{k}^{\perp}\left(\mathbf{C}^{2 N}\right)$

and $\pi(W)=W \oplus J W$.

$$
=\left\{W \in G r_{k}\left(\mathbf{C}^{2 N}\right) \mid(W, W)^{a}=0\right\}
$$

(DIII) In the case $M=S O(2 n) / U(n)=\left\{W \in G r_{n}\left(\mathbf{C}^{2 n}\right) \mid(W, W)=0\right\}$,

(1) $Z=M=S O(2 n) / U(n)$ and $\pi=i d$,

(2) $Z=\mathcal{Z}_{1, n}\left(\mathbf{C}^{2 n}\right)$

$$
=\left\{\left(W_{1}, W_{n}\right) \in G r_{1}\left(\mathbf{C}^{2 n}\right) \times G r_{n}\left(\mathbf{C}^{2 n}\right) \mid W_{1} \subset W_{n},\left(W_{n}, W_{n}\right)=0\right\}
$$
and $\pi\left(W_{1}, W_{n}\right)=W_{1} \oplus \overline{\left(W_{1}^{\perp} \cap W_{n}\right)}$.

\section{Morse-Bott theoretic deformations of harmonic maps}

The method of Morse-Bott theoretic deformations of harmonic maps under the group actions was presented in [GO1]. The natural action of complex loop group $L G \mathbf{C}$ can be used to study the space of harmonic maps. The idea is to use a one-parameter subgroup to deform a given extended solution into a simpler one. In the case at hand, the oneparameter subgroups induce the gradient flows of suitable Morse-Bott functions and so 
are quite to easy to work with. This analysis leads to results concerning the connectedness and the fundamental groups of the space of certain classes of harmonic maps.

THEOREM 4.1 [GO1]. Let $\varphi: \Sigma \longrightarrow U(n)$ be a harmonic map of finite uniton number. Assume that $\varphi$ has an extended solution $\Phi=\sum_{\alpha=0}^{m} \lambda^{\alpha} T_{\alpha}$ satisfying rank $T_{0}(z) \geqq 2$ for all $z \in \Sigma$. Then $\varphi: \Sigma \longrightarrow U(n)$ can be contınuously deformed through harmonic maps of fintte uniton number into a harmonic map $\psi: \Sigma \longrightarrow U(n-1)$ of finite uniton number.

Next we recall the notion of the Gauss bundles and the isotropy (cf. [EL]). Let $\varphi: \Sigma \longrightarrow \operatorname{Gr}\left(\mathbf{C}^{N}\right)$ be a harmonic map. Let $\underline{\varphi}$ denote the complex vector subbundle of the trivial bundle $\underline{\mathbf{C}}^{N}=\Sigma \times \mathbf{C}^{N}$ corresponding to the map $\varphi$. Then we can define the $\partial^{\prime}$-Gauss bundle $G^{\prime}(\varphi)$ as a complex subbundle induced by the image of the $\partial^{\prime}$-second fundamental form $A_{\varphi}^{\prime}=\pi_{\varphi}^{\perp} \partial: \underline{\varphi} \longrightarrow \underline{\varphi}^{\perp}$, and it corresponds to a harmonic map $\Sigma \longrightarrow$ $\operatorname{Gr}\left(\mathbf{C}^{N}\right)$. Define the $i$-th $\partial^{\prime}$-Gauss bundle $G^{(i)}(\varphi)$ of $\varphi$ by $G^{(i)}(\varphi)=G^{\prime}\left(G^{(i-1)}(\varphi)\right)$, inductively. We say that the harmonic map $\varphi$ is strongly isotropic if all $G^{(i)}(\varphi)(i \geqq 1)$ are orthogonal to $\varphi$ with respect to the Hermitian inner product of $\mathbf{C}^{N}$. A strongly isotropic harmonic map $\varphi: \Sigma \longrightarrow \mathbf{C} P^{n}=G r_{1}\left(\mathbf{C}^{N}\right)$ is called simply isotropic. Any holomorphic map of a Riemann surface into $\operatorname{Gr}\left(\mathbf{C}^{N}\right)$ is strongly isotropic. It is known (cf. [EL]) that a harmonic map of a Riemann sphere $S^{2}$ into $S^{n}, \mathbf{R} P^{n}$ or $\mathbf{C} P^{n}$ is always isotropic as a harmonic map $S^{2} \longrightarrow\left(S^{n} \longrightarrow \mathbf{R} P^{n} C\right) \mathbf{C} P^{n}$.

THEOREM 4.2 [GO1]. If $n \geqq 2$, then any ssotropıc harmonıc map $\varphi: \Sigma \longrightarrow \mathbf{C} P^{n}$ can be deformed continuously through isotropic harmonic maps into an isotropic harmonıc map $\psi: \Sigma \longrightarrow \mathbf{C} P^{2}$. In partıcular, if $n \geqq 2$, then any harmonıc map $\varphi$ : $S^{2} \longrightarrow \mathbf{C} P^{n}$ can be deformed contınuously through harmonic maps into a harmonic map $\psi: S^{2} \longrightarrow \mathbf{C} P^{2}$.

ConjeCtURE (cf. [GO1]). The space of harmonıc maps of a Rıemann sphere $S^{2}$ into $\mathbf{C} P^{n}$ with fixed energy and fixed degree is path-connected.

By Theorem 4.2, it suffices to verify this conjecture in the case $n=2$. Very recently this has been proved by T. A. Crawford [Cr].

THEOREM 4.3 [Ko],[GO1]. If $n \geqq 2$, then any isotropic harmonic map $\varphi: \Sigma \longrightarrow$ $S^{n}$ can be deformed contınuously through isotropıc harmonic maps into a holomorphic map $\psi: \Sigma \longrightarrow S^{2}$. In particular, if $n \geqq 2$, then any harmonic map $\varphi: S^{2} \longrightarrow S^{n}$ can be deformed contınuously through harmonıc maps ınto a holomorphic map $\psi: S^{2} \longrightarrow S^{2}$.

Let $\operatorname{Harm}_{k}\left(S^{2}, S^{n}\right)$ be the space of harmonic 2-spheres in the $n$-dimensional unit sphere $S^{n}$ of energy $4 \pi k$, where $k$ is a positive integer. It is well-known that if $n=2$, then $\operatorname{Harm}_{k}\left(S^{2}, S^{2}\right)$ has just two connected components consisting of the spaces of holomorphic maps of degree $k$ and anti-holomorphic maps of degree $-k$.

CoROllaRY 4.4 [Ko]. If $n \geqq 3$, then Harm $_{k}\left(S^{2}, S^{n}\right)$ is path-connected and hence Harm $_{k}\left(S^{2}, \mathbf{R} P^{n}\right)$ is also path-connected. 
In the case $n=4$ this result was proved by Loo [Lo] and independently Verdier [Ve]. Kotani [Ko] proved the above result for $n \geqq 3$.

It is known that each of $\operatorname{Harm}_{k}\left(S^{2}, S^{2}\right)$ has fundamental group $\mathbf{Z} / 2 k \mathbf{Z}$ ([Ep]). Moreover, in [FGKO] we have determined the fundamental group of the space $\operatorname{Harm}_{k}\left(S^{2}, S^{n}\right)$.

THEOREM 4.5 [FGKO].

$$
\pi_{1} \operatorname{Harm}_{k}\left(S^{2}, S^{n}\right)= \begin{cases}0 & \text { if } n \geq 4, k \neq 2 \\ \mathbf{Z} / 2 \mathbf{Z} & \text { if } n \geq 4, k=2 \\ \mathbf{Z} / 2 k \mathbf{Z} & \text { if } n=3\end{cases}
$$

There is a similar result for $\operatorname{Harm}_{k}\left(S^{2}, \mathbf{R} P^{n}\right)$ as follows.

THEOREM 4.6 [FGKO].

$$
\pi_{1} \operatorname{Harm}_{k}\left(S^{2}, \mathbf{R} P^{n}\right)= \begin{cases}\mathbf{Z} / 2 \mathbf{Z} & \text { if } n \geq 4, k \neq 2 \\ \mathbf{Z} / 2 \mathbf{Z} \oplus \mathbf{Z} / 2 \mathbf{Z} & \text { if } n \geq 4, k=2 \\ \mathbf{Z} / 2 k \mathbf{Z} \oplus \mathbf{Z} / 2 \mathbf{Z} & \text { if } n=3 \\ \mathbf{Z} / 2 k \mathbf{Z} & \text { if } n=2\end{cases}
$$

We want to mention the connectedness of the space of harmonic 2-spheres in a quaternionic projective space $\mathbf{H} P^{n}$. Bahy-El-Dien and Wood [DW2] gave the construction theory of all harmonic 2-spheres in $\mathbf{H} P^{n}$.

The quaternionic projective space $\mathbf{H} P^{n}$ has two standard twistor spaces $\mathbf{C} P^{2 n+1}$ and $\mathcal{T}_{n}([\mathrm{Br}],[\mathrm{Gl}])$. We have the identification

$$
\mathbf{H} P^{n}=\left\{V \in G r_{2}\left(\mathbf{C}^{2(n+1)}\right) \mid V=J V\right\} .
$$

The twistor space $\mathcal{T}_{n}$ over $\mathbf{H} P^{n}$ is defined

$$
\mathcal{T}_{n}=\left\{W \in G r_{n}\left(\mathbf{C}^{2(n+1)}\right) \mid(W, W)^{a}=0\right\}
$$

with the projection $\pi(W)=(W \oplus J W)^{\perp}$. The twistor space $\mathbf{C} P^{2 n+1}$ over $\mathbf{H} P^{n}$ is defined by the projection $\pi(L)=L \oplus J L$.

We know that any strongly isotropic harmonic map into $\mathbf{H} P^{n}$ can be lifted to a horizontal holomorphic map into $\mathcal{T}_{n}([\mathrm{Gl}])$, and any harmonic map into $\mathbf{H} P^{n}$ which can be lifted to a horizontal holomorphic map into $\mathbf{C} P^{2 n+1}$ is called a quaternıonıc mixed pair by [DW2]. Though any harmonic 2-sphere in $\mathbf{H} P^{n}$ which is neither strongly isotropic nor a quaternionic mixed pair can not be lifted to a horızontal holomorphic map in $\mathbf{C} P^{2 n+1}$ or $\mathcal{T}_{n}$, such a harmonic 2-sphere can be transformed to a quaternionic mixed pair by a finite number of forward and backward replacements ([DW2]). It is easily shown ([DW2]) that any harmonic 2-sphere in $\mathbf{H} P^{1}\left(=S^{4}\right)$ is always strongly isotropic or a quaternionic mixed pair.

Let $\operatorname{Harm}_{\mathcal{E}}\left(S^{2}, \mathbf{H} P^{n}\right)^{\text {st. ssot. }}$ and $\operatorname{Harm}_{\mathcal{E}}\left(S^{2}, \mathbf{H} P^{n}\right)^{q . m . p .}$ be the spaces of harmonic maps of $S^{2}$ into $\mathbf{H} P^{n}$ with fixed energy which are strongly isotropic and which are quaternionic mixed pairs, respectively. Applying the argument of [GO1] to horizontal holomorphic maps into the twistor spaces $\mathbf{C} P^{2 n+1}$ and $\mathcal{T}_{n}$ over $\mathbf{H} P^{n}$, M. Mukai (Tokyo Metropolitan Univ.) showed the following. 
THEOREM 4.7 [Mu1]. Harm $\operatorname{Ha}_{\mathcal{E}}\left(S^{2}, \mathbf{H} P^{n}\right)^{\text {st.zsot. }}$ and $\operatorname{Harm}_{\mathcal{E}}\left(S^{2}, \mathbf{H} P^{n}\right)^{\text {q.m.p. }}$ are path-connected. Moreover, the space of harmonic maps of $S^{2}$ into $\mathbf{H} P^{n}$ with fixed energy which are strongly isotropıc or quaternıonıc mixed pairs,

$$
\operatorname{Harm}_{\mathcal{E}}\left(S^{2}, \mathbf{H} P^{n}\right)^{\text {st.tsot. }} \bigcup \operatorname{Harm}_{\mathcal{E}}\left(S^{2}, \mathbf{H} P^{n}\right)^{\text {q.m.p. }},
$$

is also path-connected.

Problem. Is it true that the space $\operatorname{Harm}_{\mathcal{E}}\left(S^{2}, \mathbf{H} P^{n}\right)$ of all harmonic 2-spheres in $\mathbf{H} P^{n}$ of fixed energy is path-connected?

Furthermore, we obtained similar results for a real Grassmann manifold $G r_{2}\left(\mathbf{R}^{n+2}\right)$, a complex hyperquadric $Q_{n}(\mathbf{C})$ ([Mu2]) and more generally for compact classical symmetric spaces of inner type $([\mathrm{Mu} 3])$. Next we shall mention those results.

Let

$$
Q_{n}(\mathbf{C})=\left\{L \in \mathbf{C} P^{n+1} \mid(L, L)=0\right\}
$$

be the complex hyperquadric and $\widetilde{G r}\left(\mathbf{R}^{n+2}\right)$ be the real Grassmann manifold of oriented 2 -planes in $\mathbf{R}^{n+2}$. Then we have the natural identification $\widetilde{G r_{2}}\left(\mathbf{R}^{n+2}\right) \cong Q_{n}(\mathbf{C})$. Let $G r_{2}\left(\mathbf{R}^{n+2}\right)$ be the real Grassmann manifold of 2-planes in $\mathbf{R}^{n+2}$.

The complex hyperquadric $Q_{n}(\mathbf{C})$ has two standard twistor spaces, namely $Q_{n}(\mathbf{C})$ itself and for $n=2 m$

$$
\mathcal{Z}_{m}=\left\{W \in G r_{m}\left(\mathbf{C}^{2 m+2}\right) \mid(W, W)=0\right\}
$$

with the projection $\pi(W)=(W \oplus \bar{W})^{\perp}$.

The real Grassmann manifold $G r_{2}\left(\mathbf{R}^{n+2}\right)$ has two standard twistor spaces, namely $Q_{n}(\mathbf{C})$ with the projection $\pi(L)=L \oplus \bar{L}$ (double covering) and for $n=2 m$

$$
\mathcal{Z}_{m}=\left\{W \in G r_{m}\left(\mathbf{C}^{2 m+2}\right) \mid(W, W)=0\right\}
$$

with the projection $\pi(W)=(W \oplus \bar{W})^{\perp}$.

Bahy-El-Dien and Wood [DW1] also gave the construction theory of all harmonic 2-spheres in $Q_{n}(\mathbf{C})$ and $G r_{2}\left(\mathbf{R}^{n+2}\right)$. A harmonic map $\varphi: \Sigma \longrightarrow G r_{2}\left(\mathbf{R}^{n+2}\right)$ (C $\left.G r_{2}\left(\mathbf{C}^{n+2}\right)\right)$ is strongly isotropic if and only if $\varphi$ can be lifted to $Z_{m}$ as a horizontal holomorphic map. A harmonic map $\varphi: \Sigma \longrightarrow G r_{2}\left(\mathbf{R}^{n+2}\right)$ is called a real mıxed paır if $\varphi$ can be lifted to $Q_{n}(\mathbf{C})$ as a holomorphic map.

Since $H_{2}\left(Q_{n}(\mathbf{C}) ; \mathbf{Z}\right)=\mathbf{Z}$ for $n \geqq 3$, we can define the degree of $\varphi$ for each smooth $\operatorname{map} \varphi: S^{2} \longrightarrow Q_{n}(\mathbf{C})$. For $n \geqq 3$, let $\operatorname{Harm}_{d, \mathcal{E}}\left(S^{2}, Q_{n}(\mathbf{C})\right)^{\text {st.isot. }}$ and $H_{\text {ol }}\left(S^{2}, Q_{n}(\mathbf{C})\right)$ be the space of strongly isotropic harmonic maps of $S^{2}$ into $Q_{n}(\mathbf{C})$ with fixed energy and fixed degree and the space of holomorphic maps of $S^{2}$ into $Q_{n}(\mathrm{C})$ with fixed degree, respectively. Here we call a harmonic map $\varphi: \Sigma \longrightarrow Q_{n}(\mathbf{C})$ strongly isotropıc if $\varphi$ is strongly isotropic as a harmonic map $\varphi: \Sigma \longrightarrow\left(Q_{n}(\mathbf{C}) \longrightarrow G r_{2}\left(\mathbf{R}^{n+2}\right)\right) \subset G r_{2}\left(\mathbf{C}^{n+2}\right)$.

In the case $n=2$, since $H_{2}\left(Q_{2}(\mathbf{C}) ; \mathbf{Z}\right)=H_{2}\left(\mathbf{C} P^{1} \times \mathbf{C} P^{1} ; \mathbf{Z}\right)=\mathbf{Z} \oplus \mathbf{Z}$, we can define the bi-degree $\left(\operatorname{deg}_{\mathcal{L}}(\varphi), \operatorname{deg}_{\mathcal{W}}(\varphi)\right)$ for each smooth map $\varphi: S^{2} \longrightarrow Q_{2}(\mathbf{C})$. Let $\operatorname{Harm}_{d_{\mathcal{L}}, d_{\mathcal{W}}}\left(S^{2}, Q_{2}(\mathbf{C})\right)$ be the space of harmonic maps of $S^{2}$ into $Q_{2}(\mathbf{C})$ with fixed $\operatorname{deg}_{\mathcal{L}}(\varphi)$ and fixed $\operatorname{deg}_{\mathcal{W}}(\varphi)$.

THEOREM 4.8 .

(1) If $n \geqq 3$, the space $\operatorname{Harm}_{d, \mathcal{E}}\left(S^{2}, Q_{n}(\mathbf{C})\right)^{\text {st.isot. }}$ is path-connected. 
(2) If $n \geqq 3$, the space $\mathrm{Hol}_{d}\left(S^{2}, Q_{n}(\mathbf{C})\right)$ is path-connected.

(3) The space Harm $_{d_{\mathcal{L}}, d_{\mathcal{W}}}\left(S^{2}, Q_{2}(\mathbf{C})\right)$ is path-connceted.

For $n \geqq 3$, let $\operatorname{Harm}_{d, \mathcal{E}}\left(S^{2}, G_{2}\left(\mathbf{R}^{n+2}\right)\right)^{\text {st. } 2 \text { sot. }}$ be the space of strongly isotropic harmonic maps of $S^{2}$ into $G r_{2}\left(\mathbf{R}^{n+2}\right)$ with fixed energy and fixed $d(\varphi)$, where $d(\varphi)=$ $|\operatorname{deg}(\varphi)|$ for a lift $\varphi$ to $Q_{n}(\mathbf{C})$, and let $\operatorname{Harm}_{d}\left(S^{2}, G_{2}\left(\mathbf{R}^{n+2}\right)\right)^{r . m . p}$ the space of real mixed pairs of $S^{2}$ into $G r_{2}\left(\mathbf{R}^{n+2}\right)$ with fixed $d(\varphi)$.

Let $\operatorname{Harm}_{d_{\mathcal{L}}, d_{\mathcal{W}}, \epsilon}\left(S^{2}, G r_{2}\left(\mathbf{R}^{4}\right)\right)$ denote the space of harmonic maps $\varphi: S^{2} \longrightarrow$ $G r_{2}\left(\mathbf{R}^{4}\right)$ with fixed $d_{\mathcal{L}}(\varphi)$, fixed $d_{\mathcal{W}}(\varphi)$ and fixed sign $\epsilon(=1$ or -1$)$ of $\left(\operatorname{deg}_{\mathcal{L}}(\varphi)\right)$. $\left(\operatorname{deg}_{\mathcal{W}}(\varphi)\right)$, where $d_{\mathcal{L}}(\varphi)=\left|\operatorname{deg}_{\mathcal{L}}(\varphi)\right|$ and $d_{\mathcal{W}}(\varphi)=\left|\operatorname{deg}_{\mathcal{W}}(\varphi)\right|$ for a lift $\varphi$ of $\varphi$ to $Q_{2}(\mathbf{C})$.

THEOREM 4.9 .

(1) If $n \geqq 3$, the space $\mathrm{Harm}_{d, \mathcal{E}}\left(S^{2}, G r_{2}\left(\mathbf{R}^{n+2}\right)\right)^{\text {st.2sot. }}$ is path-connected.

(2) If $n \geqq 3$, the space $\mathrm{Harm}_{d}\left(S^{2}, G r_{2}\left(\mathbf{R}^{n+2}\right)\right)^{r . m . p .}$ is path-connected.

(3) The space $\mathrm{Harm}_{d_{\mathcal{L}}, d_{\mathcal{W}}, \epsilon}\left(S^{2}, G r_{2}\left(\mathbf{R}^{4}\right)\right)$ is path-connceted.

Let $M$ be a compact symmetric space of inner type and $Z$ be the standard twistor space of $M$ with the projection $\pi: Z \longrightarrow M$ (see Section 3). We consider the space of all harmonic map $\varphi: S^{2} \longrightarrow M$ which can be lifted to a horizontal holomorphic map into $Z$.

THEOREM 4.10 .

(1) $\mathrm{Hol}_{d}\left(S^{2}, G r_{k}\left(\mathbf{C}^{N}\right)\right)$ is path-connected for $k \geqq 2, N-k \geqq 2$.

(2) $\operatorname{Harm}_{\mathcal{E}}\left(S^{2}, \widetilde{G r}_{k}\left(\mathbf{R}^{N}\right)\right)^{(1)}$ is path-connected for $k \geqq 4, N-k \geqq 3$.

(3) $\operatorname{Harm}_{\mathcal{E}}\left(S^{2}, \widetilde{G r}_{k}\left(\mathbf{R}^{N}\right)\right)^{(2)}$ is path-connected for $k \geqq 4, N-k \geqq 4$.

(4) $\operatorname{Harm}_{\mathcal{E}}\left(S^{2}, \widetilde{G r}_{k}\left(\mathbf{R}^{N}\right)\right)^{(3)}$ is path-connected for $k \geqq 4, N-k \geqq 4$.

(5) $\mathrm{Hol}_{d}\left(S^{2}, S p(n) / U(n)\right)$ is path-connected for $n \geqq 2$.

(6) $\operatorname{Harm}_{\mathcal{E}}\left(S^{2}, G r_{k}\left(\mathbf{H}^{N}\right)\right)^{(1)}$ is path-connected for $k \geqq 2, N-k \geqq 2$.

(7) $\operatorname{Harm}_{\mathcal{E}}\left(S^{2}, G r_{k}\left(\mathbf{H}^{N}\right)\right)^{(2)}$ is path-connected for $k \geqq 2, N-k \geqq 2$.

(8) $\mathrm{Hol}_{d}\left(S^{2}, S O(2 n) / U(n)\right)$ is path-connected for $n \geqq 3$.

Here $\operatorname{Harm}_{\mathcal{E}}\left(S^{2}, \widetilde{G r}_{k}\left(\mathbf{R}^{N}\right)\right)^{(i)}$ denotes the space of all harmonic maps of $S^{2}$ into $\widetilde{G r}_{k}\left(\mathbf{R}^{N}\right)$ with fixed energy which can be lifted as horizontal holomorphic maps to the standard twistor space of $(i)$ in (BDI) of the list of Section 3, and $\operatorname{Harm}_{\mathcal{E}}\left(S^{2}\right.$, $\left.G r_{k}\left(\mathbf{H}^{N}\right)\right)^{(i)}$ denotes the space of all harmonic maps of $S^{2}$ into $G r_{k}\left(\mathbf{H}^{N}\right)$ with fixed energy which can be lifted as horizontal holomorphic maps to the standard twistor space of $(i)$ in (CII) of the list of Section 3 .

Remark.

(1) In the case (AIII)-(2) : $\varphi$ can be deformed continuously through harmonic maps into a (strongly isotropic) harmonic map into $G r_{k}\left(\mathbf{C}^{k+2}\right)$.

(2) In the case (AIII)-(3) : $\varphi$ can be deformed continuously through harmonic maps into a harmonic map (mixed pair) into $G r_{2}\left(\mathbf{C}^{N-k+2}\right)$.

(3) In the case (DIII)-(2) . Our argument using Morse-Bott theoretic deformations to a subtwistor space does not work. 
In a forthcoming paper, we will discuss these works in detail including results on fundamental groups of these spaces of harmonic 2-spheres.

\section{REFERENCES}

[BED-W1] A. Bahy-El-Dien and J.C.Wood, The explicit construction of all harmonic two-spheres in $G r_{2}\left(\mathbf{R}^{n}\right)$, J. Reine Angew.Math. 398 (1989), 36-66.

[BED-W2] A. Bahy-El-Dien and J.C.Wood, The explicit construction of all harmonic two-spheres in quaternionic projective spaces, Proc. London Math. Soc. (3) 62 (1991), 202-224.

[BG] M.J. Bergvelt and M.A. Guest, Actions of loop groups on harmonic maps, Trans. Amer. Math. Soc. 326 (1991), 861-886.

[Br] R.L. Bryant, Lie groups and twistor spaces, Duke Math. J. 52 (1985), 223-261.

[BR] F.E. Burstall and J.H. Rawnsley, Twistor theory for Riemannian symmetric spaces, Lecture Notes in Math. 1424, Springer-Verlag, Berlin, Heidelberg, New York, Tokyo, 1990.

[Cr] T.A. Crawford, The space of harmonic maps from the 2-sphere to complex projective space, preprint, McGill University, 1993.

[EL] J. Eells and L. Lemaire, Another report on harmonic maps, Bull. Lond. Math. Soc. 20 (1988), 385-524.

[Ep] S.I. Épshtein, Fundamental groups of spaces of coprime polynomials, Funct. Anal. Appl. 7 (1973), 82-83.

[FGKO] M. Furuta, M.A. Guest, M. Kotani and Y.Ohnita, On the fundamental group of the space of harmonic 2-spheres in the n-sphere, Math. Z. 215 (1994), 503-518.

[Gl] J. F. Glazebrook, The construction of a class of harmonic maps to quaternionic projective spaces, J. London Math. Soc. (2) 30 (1984), 151-159.

[GO1] M.A. Guest and Y.Ohnita, Group actions and deformations for harmonic maps, J. Math. Soc. Japan. 45 (1993), 671-704.

[GO2] M.A. Guest and Y.Ohnita, Loop group actions on harmonic maps and their applications, Harmonic Maps and Integrable Systems, edited by J.C. Wood and A. Fordy, Vieweg, 1994, pp. 273-292.

[Kt] M. Kotani, Connectedness of the space of minimal 2-spheres in $S^{2 m}(1)$, Proc. Amer. Math. Soc. 120 (1994), 803-810.

[Lo] B. Loo, The space of harmonic maps of $S^{2}$ into $S^{4}$, Trans. Amer. Math. Soc. 313 (1989), 81-102.

[Mu1] M. Mukai, On connectedness of the space of harmonic 2-spheres in quaternionic projective spaces, Tokyo J. Math. 17 (1994), 241-252.

[Mu2] M. Mukai, On connectedness of the space of harmonic 2-spheres in real Grassmann manifolds of 2-planes, Natur. Sci. Rep. Ochanomizu Univ. 44 (1993), 99-115.

[Mu3] M. Mukai, On the connectedness of spaces of harmonic 2-spheres, master thesis, Ochanomizu Univ., 1994.

[Po] K. Pohlmeyer, Integrable Hamiltonian systems and interactions through quadratic constraints, Commun. Math. Phys. 46 (1976), 207-221.

[PS] A.N. Pressley and G.B. Segal, Loop Groups, 1986, Oxford University Press.

[Ra] J. Rawnsley, f-strucures, f-twistor spaces and harmonic maps, Geometry Seminar "Luigi Bianchi "II-1984, Lecture Notes in Math. 1164, Springer-Verlag, Berlin, Heidelberg, New York, Tokyo, 1986, pp. 85-159.

[Sa] S.M. Salamon, Harmonic and holomorphic maps, Geometry Seminar "Luigi Bianchi" II-1984, Lecture Notes in Math. 1164, Springer-Verlag, Berlin, Heidelberg, New York, Tokyo, 1986, pp. 161-224.

[Se] G.B. Segal, Loop groups and harmonic maps, Advances in Homotopy Theory, L.M.S. Lecture Notes 139, Cambridge Univ. Press, 1989, pp. 153-164. 
[Uh] K.K. Uhlenbeck, Harmonic maps into Lie groups (Classical solutions of the chiral model), J. Differential Geom. 30 (1989), 1-50.

[Ve] J.L. Verdier, Applications harmoniques de $S^{2}$ dans $S^{4}$ :II, Harmonic Mappings, Twistors, and $\sigma$-models, Advanced Series in Math. Phys. 4 (P. Gauduchon, ed.), World Scientific (Singapore), 1988, pp. 124-147.

[ZM] V.E. Zakharov and A.V. Mikhailov, Relativistically invariant two-dimensional models of field theory which are integrable by means of the inverse scattering problem method, Sov. Phys. JETP 47 (1978), 124-147.

[ZS] V.E. Zakharov and A.B. Shabat, Integration of non-linear equations of mathematical physics by the method of inverse scattering II, Funct. Anal. Appl. 13 (1979), 13-22.

DEPARTMENT OF MATHEMATICS

TOKYo METROPOLITAN UNIVERSity

Minami-OHSAWA $1-1$, HaChIOJI

TOKYO 192-03, JAPAN 